In Situ

Revue des patrimoines
In Situ

Revue des patrimoines

$6 \mid 2005$

Patrimoine en situation : l'Inventaire général entre histoire et prospective

\title{
Les parcs des porcelainiers Haviland
}

\section{Colette Chabrely}

\section{(2) OpenEdition}

Journals

Édition électronique

URL : http://journals.openedition.org/insitu/8376

DOI : 10.4000/insitu.8376

ISSN : 1630-7305

Éditeur

Ministère de la Culture

Référence électronique

Colette Chabrely, «Les parcs des porcelainiers Haviland », In Situ [En ligne], 6 | 2005, mis en ligne le 18 avril 2012, consulté le 14 novembre 2019. URL : http://journals.openedition.org/insitu/8376 ; DOI :

10.4000/insitu.8376

Ce document a été généré automatiquement le 14 novembre 2019.

\section{(c) (i) $\odot$}

In Situ Revues des patrimoines est mis à disposition selon les termes de la licence Creative Commons Attribution - Pas d'Utilisation Commerciale - Pas de Modification 4.0 International. 


\title{
Les parcs des porcelainiers Haviland
}

\author{
Colette Chabrely
}

1 Les parcs du Reynou et de Mont-Méry en Limousin, anciennes propriétés des Haviland, présentent l'un et l'autre un grand intérêt paysager et botanique. Ces deux domaines, résidences de campagne de Charles et Théodore Haviland ${ }^{1}$, sont les témoins de leurs sensibilités respectives et du mode de vie de grands industriels de la porcelaine à la fin $\mathrm{du} \mathrm{XIX}^{\mathrm{e}}$ siècle $^{2}$. Ces parcs, à travers leurs différences, présentent des caractères d'exception. Ils sont cependant peu documentés; il s'agit ici de faire le point sur les éléments permettant de retracer leur histoire et de poser de nouveaux jalons de recherche autour de la question de leur attribution qui reste conjecturale.

\section{Le parc du Reynou ${ }^{3}$}

«On apercevait, entre les piliers de fer, des terrasses sablées, des massifs de roses de Bengale, des balustrades de granite, des arbres, des pelouses qui rejoignaient des prairies en pente, dressées contre le proche horizon et où descendaient le soir, sur de petites roues, des charrettes de foin très basses, traînées par des vaches. " Jacques Chardonne $^{4}$, à travers ces lignes écrites à propos du parc de Joncherolles dans les Destinées sentimentales ${ }^{5}$, évoque en réalité la terrasse du château du Reynou où se mêlent senteurs d'Orient et paysage limousin. Les terrasses sont propices aux évocations (fig. $\mathbf{n}^{\circ} \mathbf{1}$ ). 
Figure 1

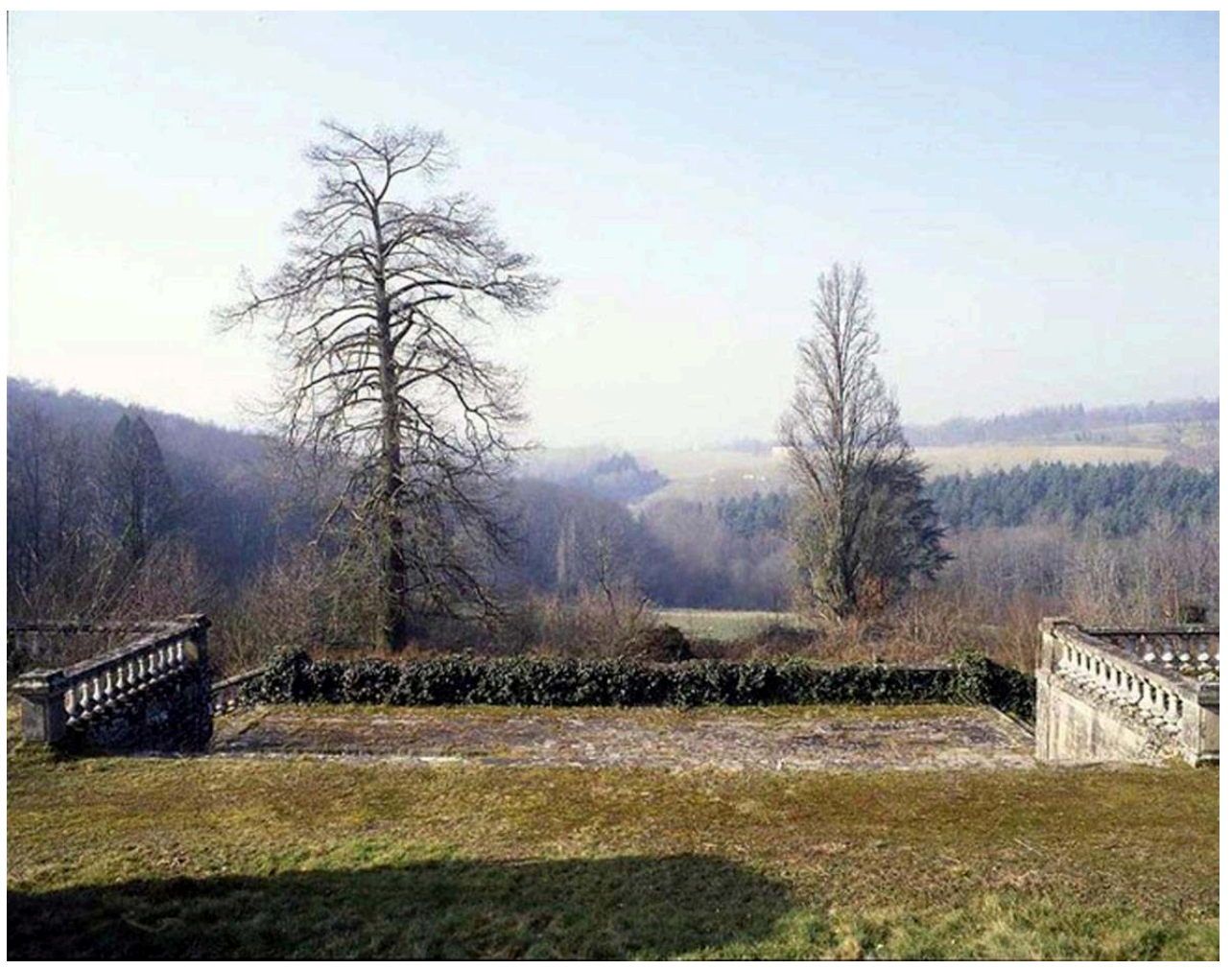

Le Reynou. Vue de la terrasse sud-ouest prise à partir de la véranda. Cette photo de 1993 montre les deux arbres (taxodium distichum, quercus robur fastigiata) qui encadraient la vue sur le bocage agricole Phot. Inv. P. Rivière @ Inventaire général, ADAGP, 1993

3 Situé sur la partie supérieure d'un vallon au creux duquel coule un petit ruisseau, ce parc est composé d'une succession de scènes. L'allée principale est bordée d'un côté d'une prairie plantée, de l'autre d'une rivière anglaise. Dans la perspective de la façade postérieure du château se dresse un tertre figurant une scène de montagne ornée de conifères. Devant la façade sud-ouest, une terrasse dominant la rivière anglaise et son île offre une vue sur la campagne vallonnée, cadrée par deux arbres remarquables. Enfin, une seconde terrasse de niveau, au sud du château, conduit à une série de trois pièces d'eau bordées d'un ensemble exceptionnel de rocailles où se succèdent pont rustique, faux enrochements, grotte, rochers en équilibre. Un belvédère permettant la vue sur les pièces d'eau et le château présente un étonnant garde-corps formé d'un arbre artificiel. L'ensemble est une parfaite illustration de tous les thèmes proposés par Edouard André dans son Traité général de la composition des parcs et jardins ${ }^{6}$. Ces espaces d'agrément sont complétés d'un jardin de fleurs ${ }^{7}$, d'un jardin potager, d'un verger. Ils sont prolongés par quelques allées cavalières, situées aujourd'hui en-dehors de la propriété.

En l'absence de sources documentaires, c'est la chronologie des différents travaux d'agrandissement du château ${ }^{8}$, elle-même rapprochée de ce que nous savons de la biographie de Charles Haviland ${ }^{9}$ et, notamment, de sa réussite professionnelle, qui permet de proposer une datation du $\operatorname{parc}^{10}$ (fig. $n^{\circ} 2$ ). 
Figure 2

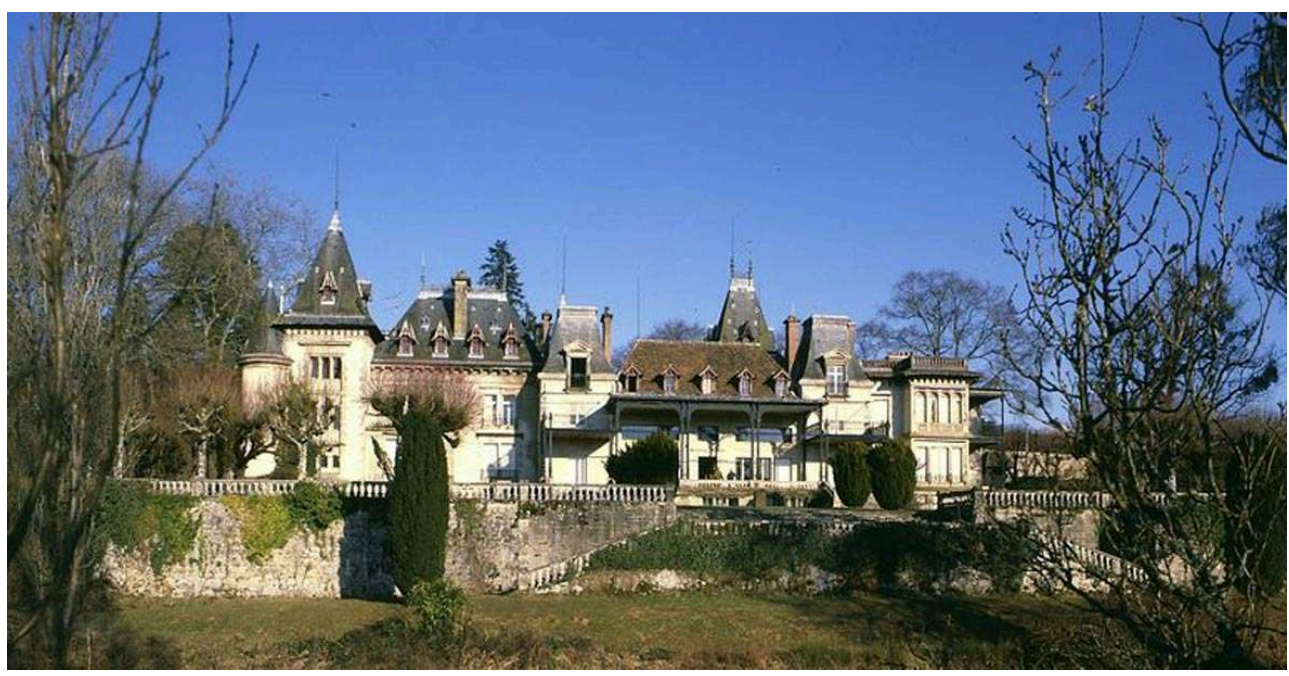

Le Reynou. Vue générale de la façade sud-ouest prise avant la réfection de la couverture

Phot. Inv. P. Rivière @ Inventaire général, ADAGP, 1991

\section{Une création en plusieurs étapes}

5 Deux photographies ${ }^{11}$ du parc prises à partir des pièces d'eau en direction du château montrent la silhouette de la demeure pourvue des tours qui lui furent ajoutées en 1877 mais sans les pavillons construits en 1894-95 (fig. $\mathbf{n}^{\circ}$ 3). Sur ces vues, la terrasse sud dite autrefois terrasse des platanes présente des arbres en alignement, de belle taille sans être énormes, alors que les arbres plantés entre l'escalier situé en contrebas et les pièces d'eau sont des sujets jeunes dont certains sont encore pourvus de leurs tuteurs de plantation. Il semble donc que les aménagements du parc auraient été réalisés au moins en deux campagnes de travaux. La plus ancienne pourrait correspondre à l'accès au domaine et aux deux terrasses sud-ouest et sud. L'aménagement et la plantation de la terrasse des platanes pourrait aller de pair avec les premiers agrandissements du château autour de 1877-80. La seconde concernerait la création des spectaculaires pièces d'eau avec toutes les rocailles et se situerait peu avant 1893, date à laquelle des photographies les montrent nouvellement réalisées, sans doute à peine achevées. 


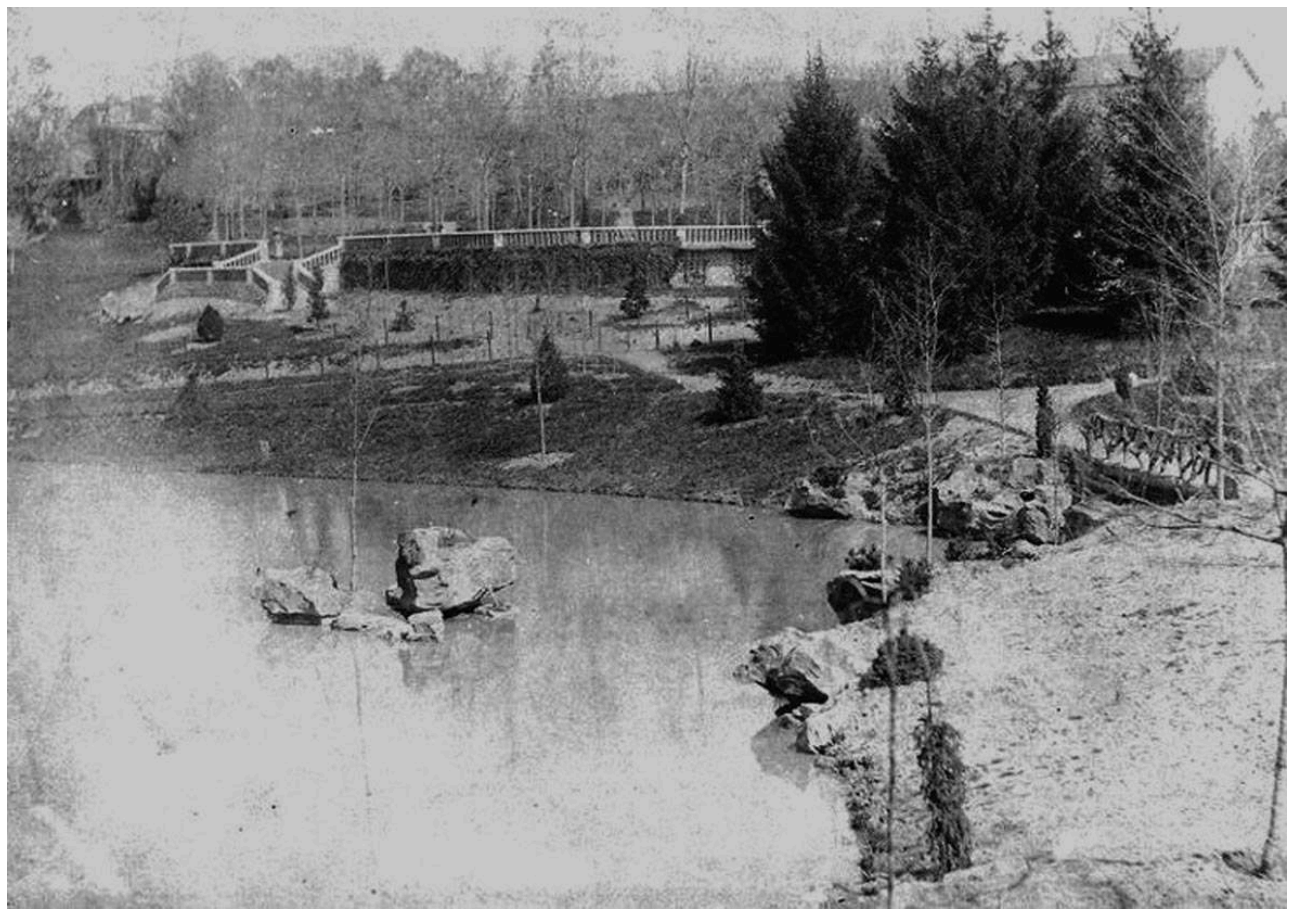

Le Reynou. Vue des pièces d'eau nouvellement créées prise en direction du château. Ce document montre la terrasse sud plantée et des sujets encore très petits en contrebas de cette dernière, ce qui conduit à penser qu'il y a eu plusieurs phases d'aménagement. Le château, au second plan, se présente sans les pavillons nord et sud, ce qui situe ce document antérieurement à 1894-95 et date l'aménagement des pièces d'eau d'avant ces ajouts

Phot. Inv. P. Rivière @ Inventaire général, ADAGP, 1992

6 La construction de l'aile méridionale du château projetée en 1894 serait alors consécutive à ces derniers aménagements du parc et probablement liée à ceux-ci. Le parti architectural vénitien et néo-colonial de ce corps de bâtiment offre l'avantage d'être très ouvert, ménageant de belles vues vers le sud, partie alors nouvelle et spectaculaire du parc. Dans l'état actuel, l'obstruction des échappées visuelles du fait de la croissance incontrôlée de la végétation efface progressivement les idées directrices qui ont présidé à tous ces aménagements. Les photographies anciennes des rocailles témoignent de leur impact visuel avant que la patine et la végétation ne les intègrent au paysage, et ne les rendent en parfaite symbiose avec la «vraie nature » (fig. $\mathbf{n}^{\circ} \mathbf{4}$ ) (fig. $n^{\circ}$ 5). 
Figure 4

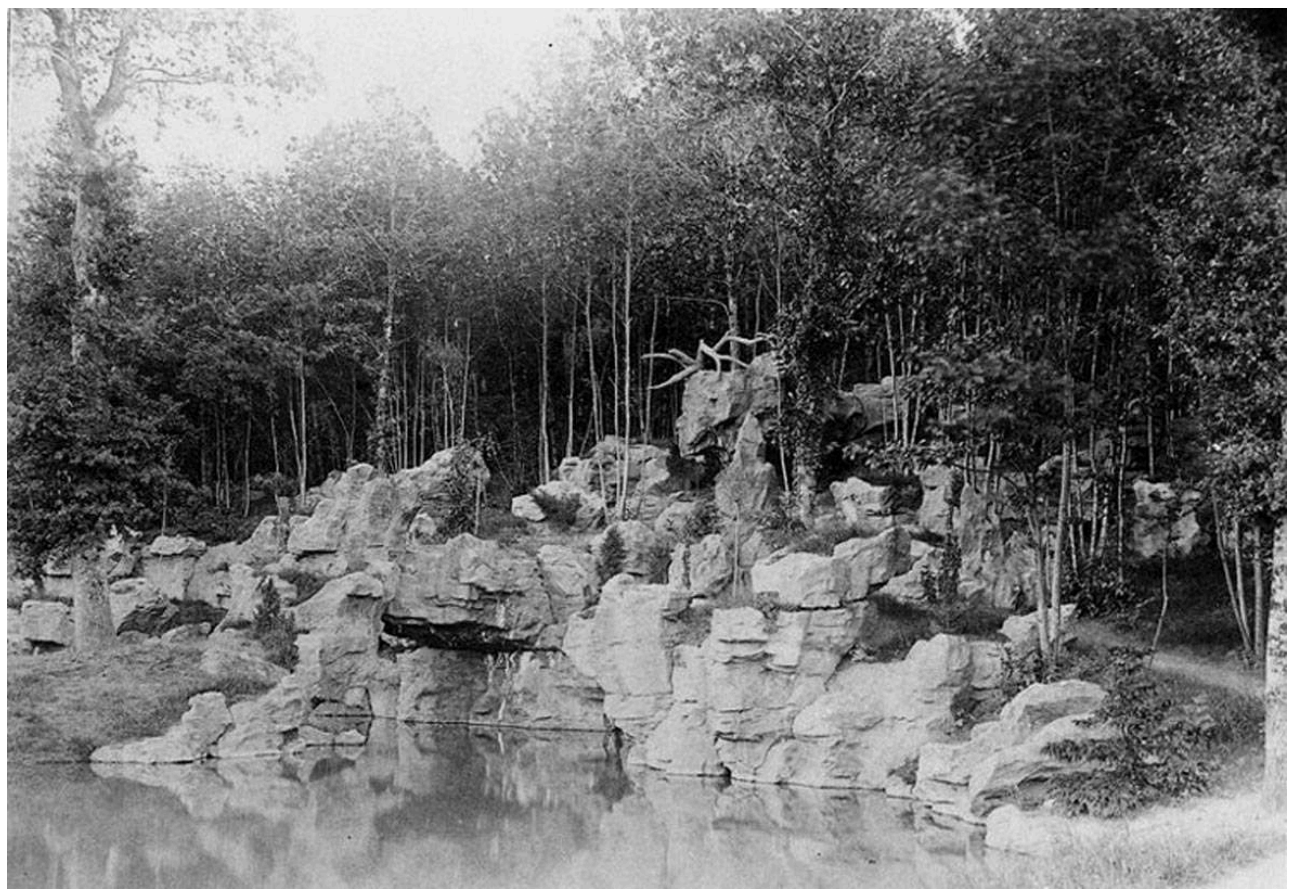

Le Reynou. Vue des rocailles autour des pièces d'eau, détail de l'arc de rochers et du belvédère avec son garde-corps en forme de branches d'arbres

Phot. Inv. P. Rivière @ Inventaire général, ADAGP, 1992

\section{Figure 5}

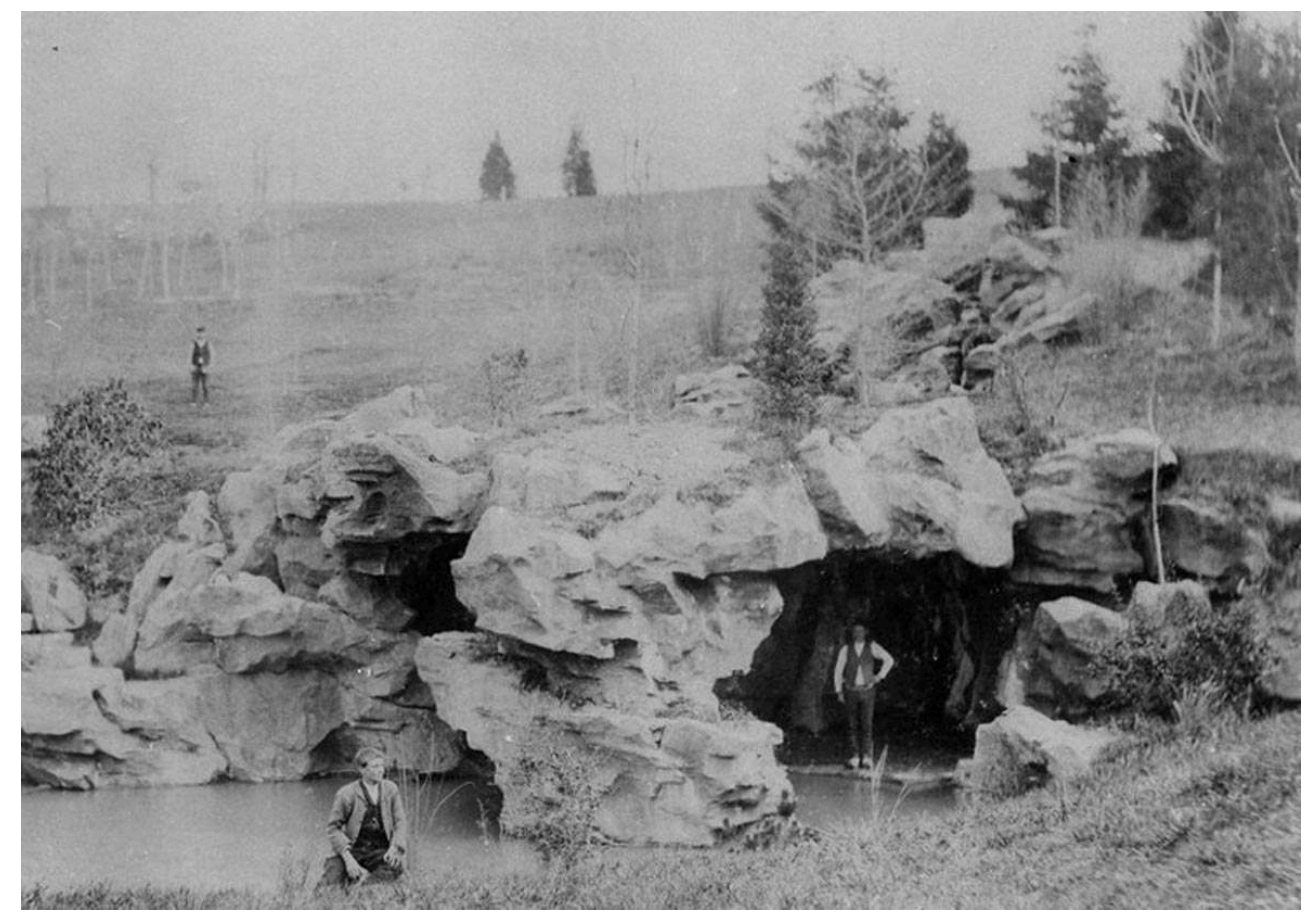

Le Reynou. Vue de la grotte artificielle et de la cascade au second plan Phot. Inv. P. Rivière @ Inventaire général, ADAGP, 1997 
7 D'autres photos prises lors des travaux d'agrandissement du château montrent que des statues disposées près de la construction ont été protégées pendant le temps du chantier (fig. $\mathbf{n}^{\circ}$ 6) (fig. $\mathbf{n}^{\circ}$ 7). Charles Haviland, collectionneur ${ }^{12}$, n'avait pas manqué en effet de disposer des œuvres à différents endroits du parc. Une de ces photos anciennes montre des personnages de pierre disposés sur la balustrade de la terrasse sud.Les dommages du temps, les tempêtes, le manque d'entretien, les périodes de déshérence, ainsi que les fréquents changements de propriétaires, ont insensiblement éloigné ce parc de son état d'origine, brouillant la lecture de cette œuvre et faussant la perception des effets imaginés par les concepteurs.

Figure 6

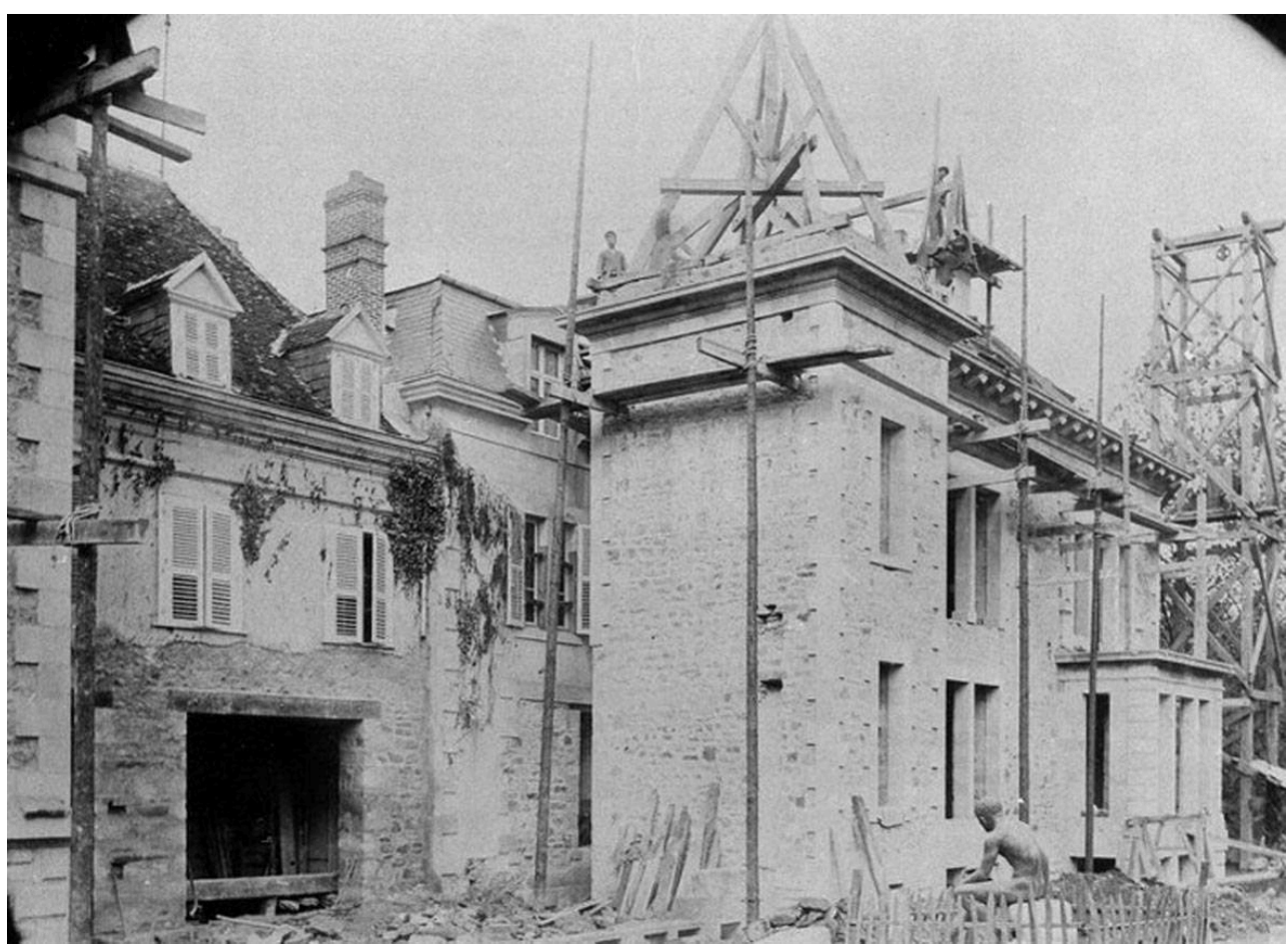

Le Reynou. Vue du chantier de construction de l'aile nord. Au premier plan une sculpture d'un personnage grandeur nature assis est protégée par une palissade

Phot. Inv. P. Rivière @ Inventaire général, ADAGP, 1992 


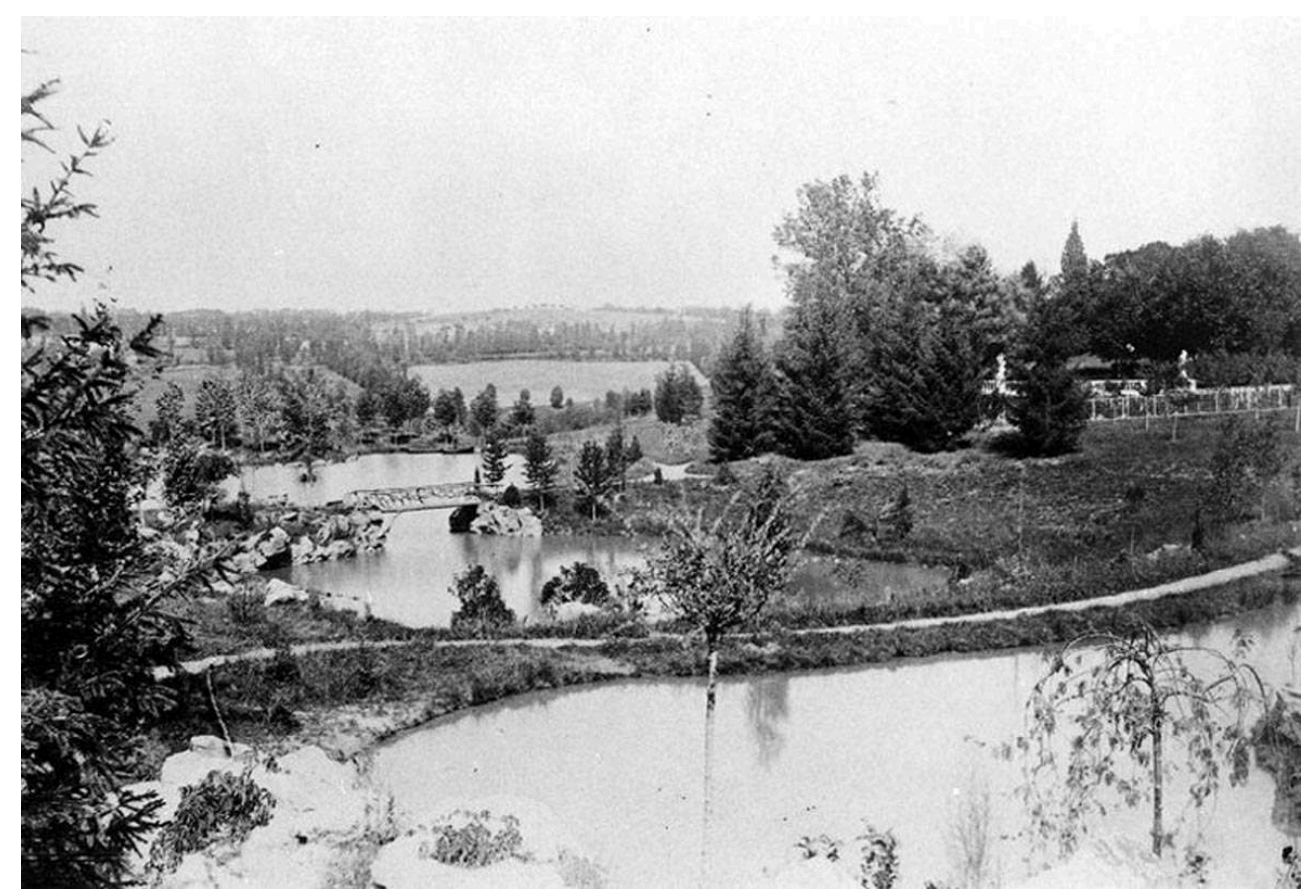

Le Reynou. Vue des trois pièces d'eau nouvellement créées. On distingue au second plan les sculptures disposées sur la balustrade de la terrasse

Phot. Inv. P. Rivière @ Inventaire général, ADAGP, 1992

\section{Quel(s) créateur(s) pour le parc du Reynou ?}

8 Ce parc exceptionnel est vraisemblablement l'œuvre du pépiniériste paysagiste André Laurent ${ }^{13}$, sur lequel on sait peu de choses hors les indications données par sa nécrologie ${ }^{14}$ qui lui attribue quatre parcs : Plantadis ${ }^{15}$, Crochat $^{16}$, le Reynou et MontMéry. De toute évidence, ces deux derniers étant les parcs les plus exceptionnels autour de Limoges, une contribution à ces réalisations pouvait éventuellement valoir attribution à l'heure des reconnaissances ultimes. Pour le Reynou un deuxième texte vient conforter cette affirmation; il s'agit d'un compte rendu de l'«excursion printanière » effectuée dans ce parc en 1931 par l'association des « Amis des fleurs » qui exprime l'enchantement des visiteurs et précise que « ce parc a été créé il y a près d'un demi-siècle par le maître-paysagiste André Laurent $\aleph^{17}$. Mais aucun plan, devis ou facture ne vient confirmer ni préciser la contribution de ce praticien.

9 La fourchette chronologique des travaux du Reynou se situe dans la période où André Laurent jouit d'une certaine renommée. Charles Haviland qui a fait travailler l'entrepreneur limougeaud Rouveroux pour les agrandissements de la demeure, a fort bien pu confier à André Laurent, principal homme de l'art local, sinon la conception du parc, du moins sa réalisation et la fourniture des végétaux. Seule une analyse fine de la composition et des végétaux, comparée à celle d'autres parcs signés par ce paysagiste permettrait de cerner le rôle exact qu'il joua au Reynou et, par la même occasion, la part prise par le commanditaire.

10 Les pièces d'eau et l'ensemble des éléments rustiques reviennent à l'entrepreneur de rocailles Gabriel Lecardeur comme l'a démontré Michel Racine ${ }^{18}$. Le pont rustique est 
parfaitement semblable par des détails significatifs au pont représenté sur une gravure publicitaire de cette entreprise. Une jambe de force située sous le tablier prenant appui sur les rochers de la berge est un détail d'assemblage qui peut confirmer cette attribution. Toute cette partie aquatique pourrait être la complète création de Gabriel Lecardeur, intervenant à la fois comme concepteur et comme fournisseur des éléments rustiques (fig. $\mathbf{n}^{\circ} \mathbf{8}$ ).

Figure 8

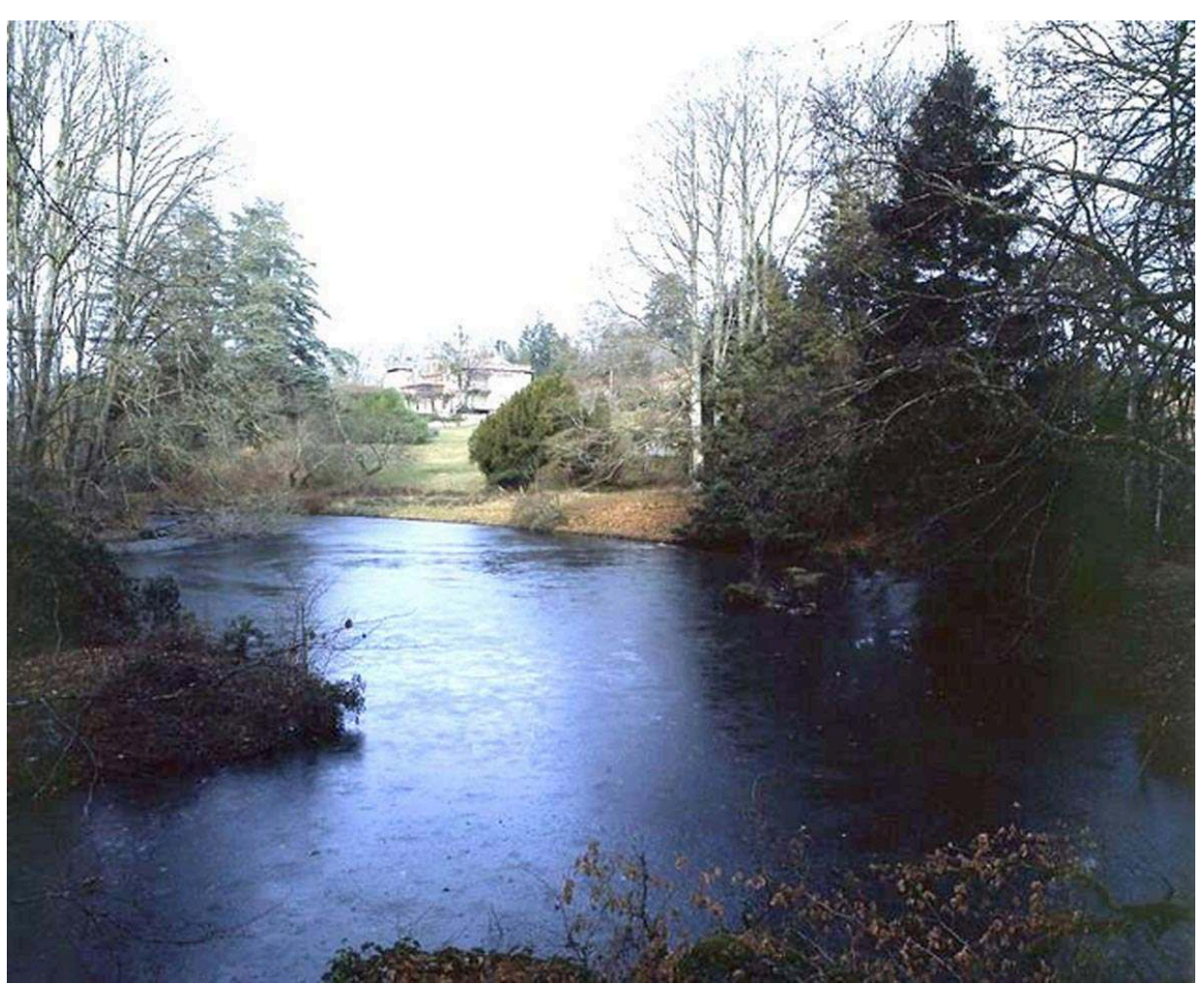

Le Reynou. Vue générale à partir des pièces d'eau, en direction du château

Phot. Inv. P. Rivière @ Inventaire général, ADAGP, 1991

\section{Le parc de Mont-Méry ${ }^{19}$}

A Mont-Méry, devenu Beaubatou sous la plume de J. Chardonne, « on mange du maïs vert, des cèpes à la crème, du lait caillé. Quand il pleut, les grands horizons se couvrent de brume et les sous-bois sont parfumés » (fig. $\mathbf{n}^{\circ}$ 9). La terre de Mont-Méry est bien celle qui convient aux cèpes, mais manger du maïs vert n'était pas l'usage dans les monts d'Ambazac sauf à adopter des coutumes venues d'ailleurs, par exemple des EtatsUnis où Théodore Haviland ${ }^{20}$ avait passé plusieurs années et épousé Julie Danat, une jeune Américaine, avant de revenir à Limoges en 1879. En 1883, il achète la terre de Moncocu située sur une émergence granitique culminant à 585 mètres, le mont Gerbassou au cœur des monts d'Ambazac. De là, en direction de l'est et du sud, se découvre une vue exceptionnelle vers des horizons le plus souvent voilés, bleutés, violets parfois, de cette couleur encrée qui caractérise les lointains limousins. La terre de Moncocu a été choisie pour le site qu'elle offre. Elle fut joliment rebaptisée MontMéry. 
Figure 9

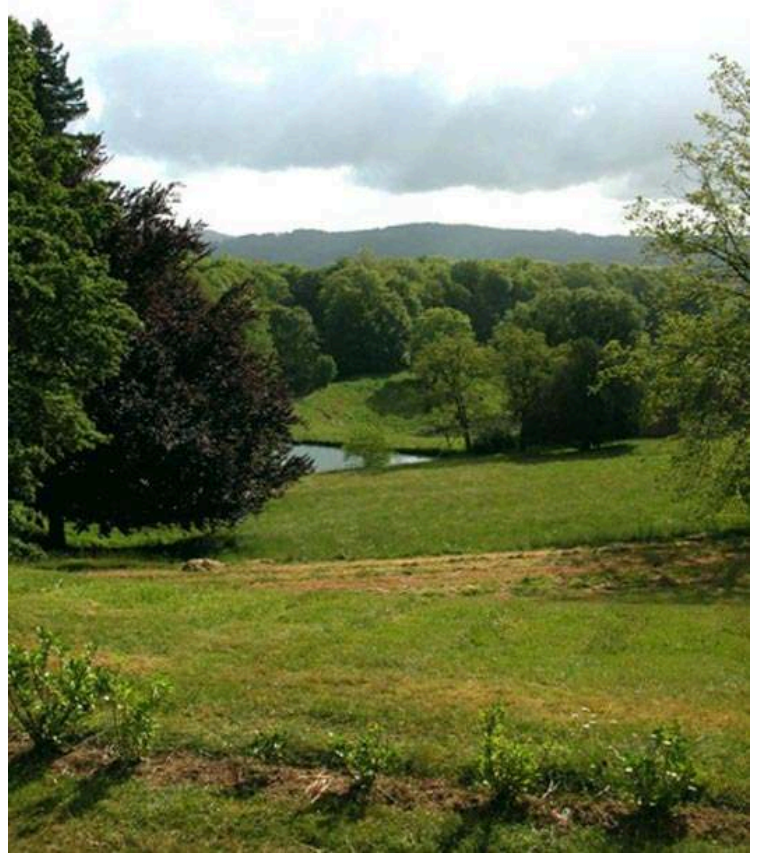

Mont-Méry. La terre de Mont-Méry dans les monts d'Ambazac Phot. Inv. J.-L. Vey (C) Inventaire général, ADAGP, 2005

12 Le château situé sur le flanc sud et est du Gerbassou, porte le monogramme de Théodore Haviland et la date d'achèvement de la construction, $1889^{21}$.

13 L'arrivée au domaine se faisait par une majestueuse allée de hêtres et de chênes en harmonie avec la campagne limousine (cette allée a été presque entièrement dévastée par la tempête de 1999 alors que le reste du parc a été épargné). 
Figure 10

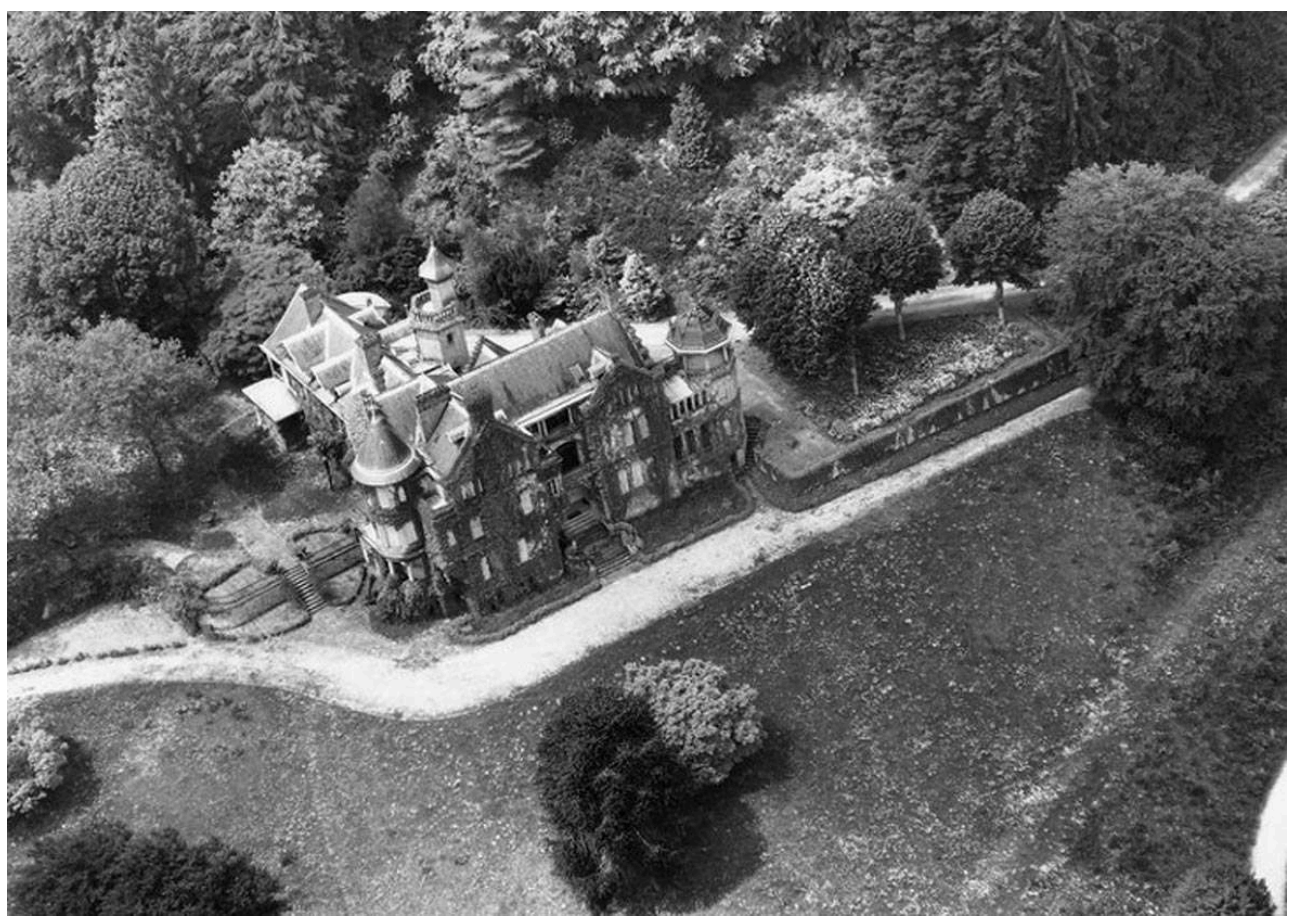

Mont-Méry. Vue aérienne prise du sud-est

Phot. Inv. R. Henrard @ Inventaire général, ADAGP, 1955

C'est au bout de cette longue voie d'accès, après que l'on ait franchi le portail

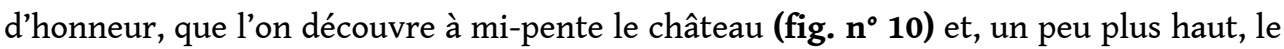
bâtiment d'écurie bien dessiné, construction agréablement mise en scène dans cet espace paysager. Entre le portail et le château monte une vaste pelouse au modelé souple, plantée d'essences rares (fig. $\mathbf{n}^{\circ} \mathbf{1 1}$ ). Ces arbres ou arbustes ayant atteint un développement exceptionnel, offrent une composition qui n'a pas son équivalent dans les autres parcs paysagers créés dans la région aux mêmes dates. Le chemin conduisant au château réserve d'autres surprises faites d'ombre, sous la ramure des grands cèdres, ou de taches de lumière à travers les troncs des andromèdes centenaires. Un petit chalet en rondins rustiques, traité comme une fabrique, porte le nom merveilleux de 《Fairy Lodge $~^{22}$. La partie supérieure du Gerbassou était sillonnée d'allées cavalières, l'une d'elles, de forme oblongue servait d'hippodrome. 
Figure 11

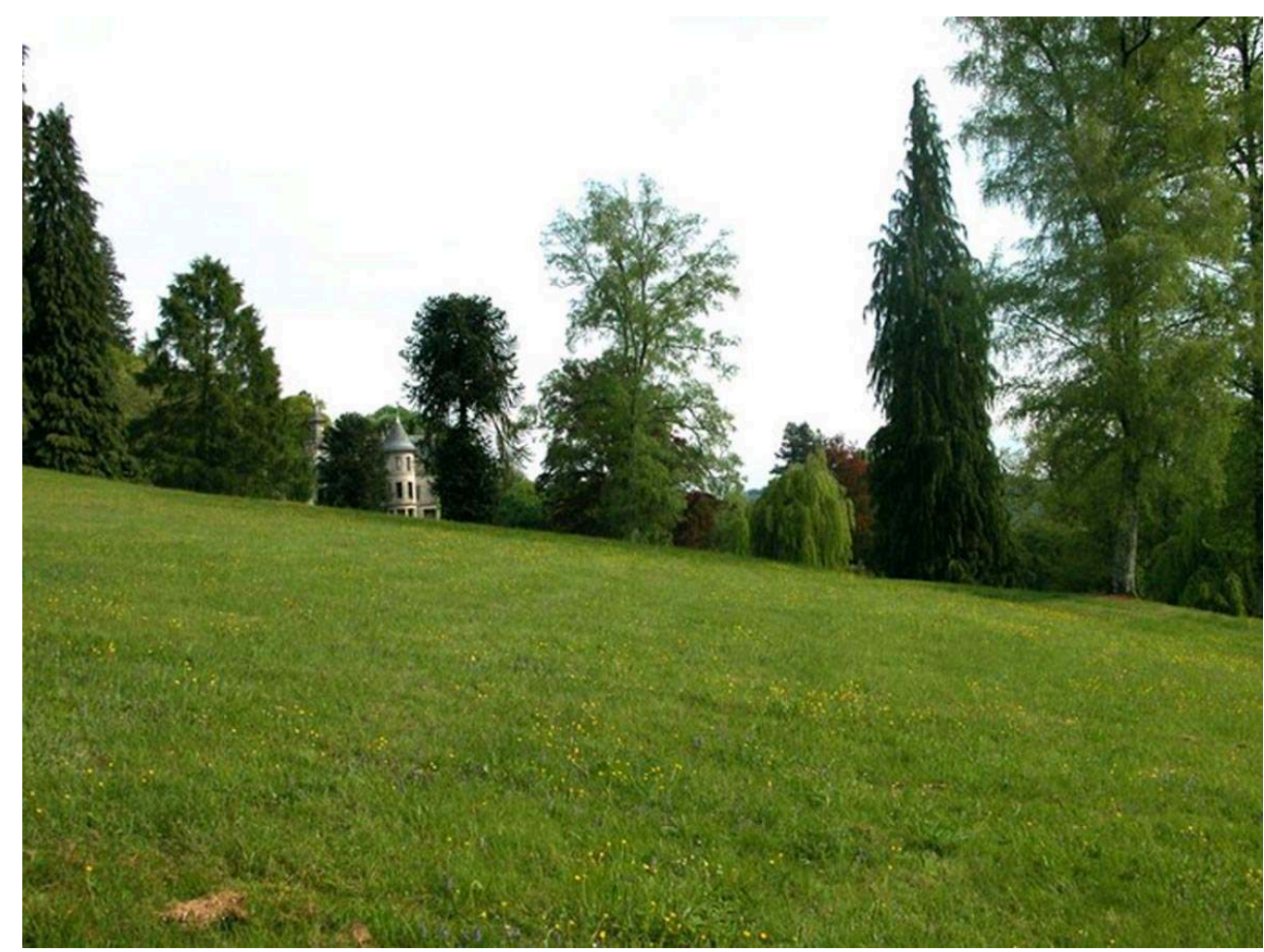

Mont-Méry. Vue éloignée prise du sud, le château et sa collection d'arbres remarquables

Phot. Inv. J.-L. Vey @ Inventaire général, ADAGP, 2005

Ce parc est un projet global, sans effet ostentatoire. Aménagé dans un site vallonné, planté de nombreux grands conifères mêlés à des feuillus d'une palette végétale très colorée en automne, il semble avoir été conçu pour recréer l'atmosphère des grands espaces américains dont Théodore et son épouse pouvaient avoir la nostalgie. Château et parc entretiennent une relation en tous points comparable à celle qui s'établit dans les constructions nord-américaines largement ouvertes sur la nature et réalisées dans des sites remarquables pour de riches commanditaires (fig. $\mathbf{n}^{\circ} \mathbf{1 2}$ ). 
Figure 12

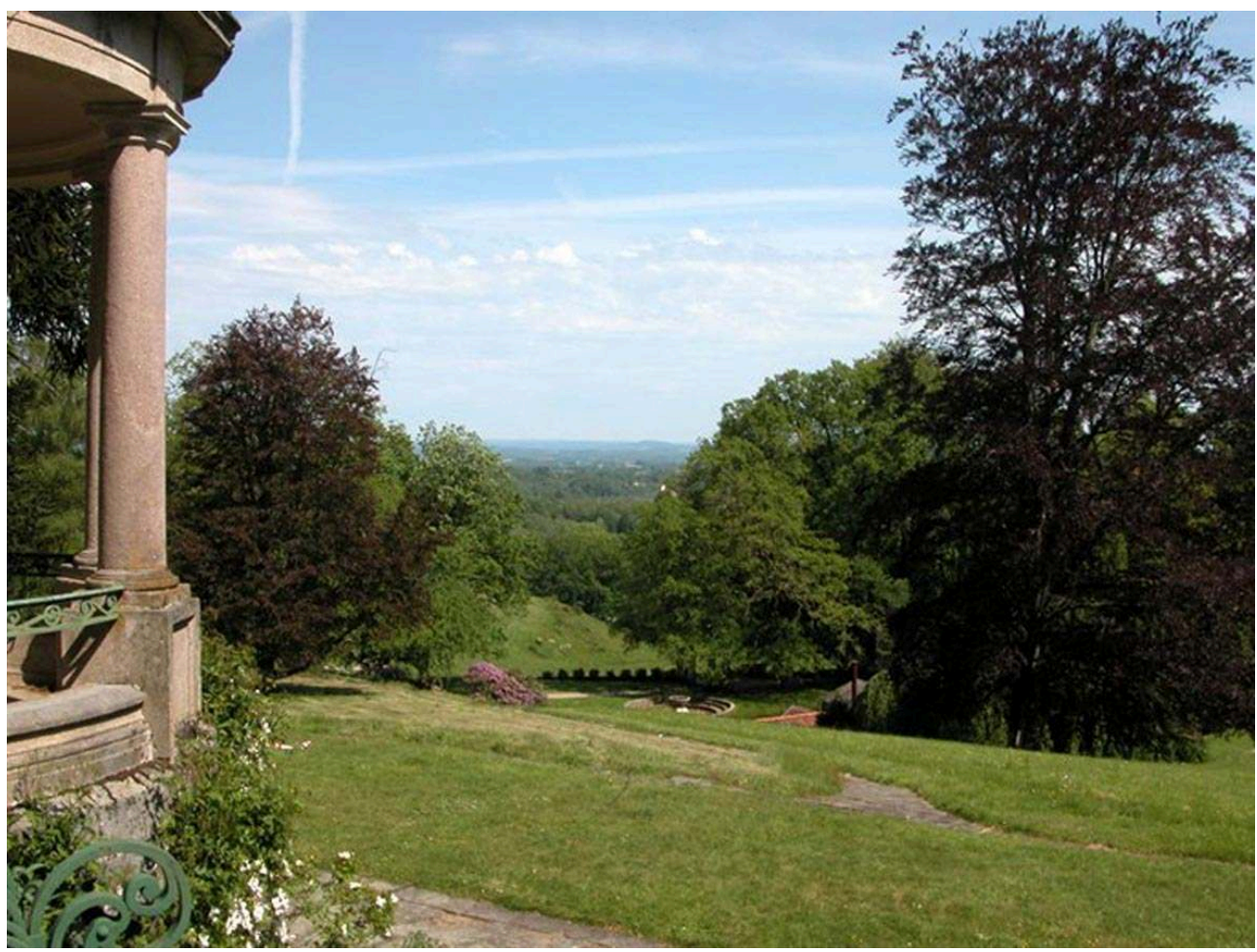

Mont-Méry. Vue des lointains vers l'est à partir de la terrasse sud

Phot. Inv. J.-L. Vey @ Inventaire général, ADAGP, 2005

\section{Quel paysagiste pour le parc de Mont-Méry?}

Une facture ${ }^{23}$ des « Grandes pépinières du Centre, André Laurent et Compagnie dont le siège est avenue de Louyat à Limoges " adressée à Théodore Haviland au château de Mont-Méry et datée de juin 1890 témoigne de la participation de ce praticien comme fournisseur de végétaux et exécutant. Ce document récapitule différentes livraisons entre janvier et juin ainsi qu'un report de facture de décembre 1889 pour une livraison de " pierre pour rocher ». Cette date confirme le fait que château et parc forment un unique projet. Une seconde liste d'arbres et arbustes des pépinières Chouve et Fils porte le nom de Théodore Haviland. Ce document non daté, pourrait être un devis plutôt qu'une facture.

De précédents travaux ont attribué ce parc à Edouard André mais, à notre connaissance, sans preuve documentaire, même si le nom de ce grand paysagiste est resté dans la mémoire des descendants de Théodore Haviland. Les nombreux travaux publiés ces dernières années autour de cet artiste ne font d'ailleurs aucun cas du parc de Mont-Méry ni de son prestigieux commanditaire ${ }^{24}$.

En revanche, les recherches sur Edouard André publiées en $2001^{25}$ mettent en évidence les liens qu'il y avait entre ce dernier et deux Américains, le botaniste Charles Sprague Sargent, directeur de l'arboretum Arnold à Jamaica Plain (un quartier de Boston), et l'architecte paysagiste Frederick Law Olmsted, créateur de Central Park à New-York ${ }^{26}$. Il y a une vraie convergence des hommes et des idées sur la recherche de nouvelles plantes, la constitution de collections et les créations paysagères. Durant son séjour 
américain dans les années 1872-75, Théodore Haviland a pu être en contact avec ce milieu et séduit par les collections de plantes rassemblées par Sargent. On trouve à Mont-Méry une collection d'essences rares (par exemple, zelkova, magnolia macrophylla, kalmia blanc...) qui semblent avoir été importées spécifiquement pour ce parc comme l'ont fait apparaître des travaux précédents ${ }^{27}$ (fig. $\mathbf{n}^{\circ} \mathbf{1 3}$ ).

Figure 13

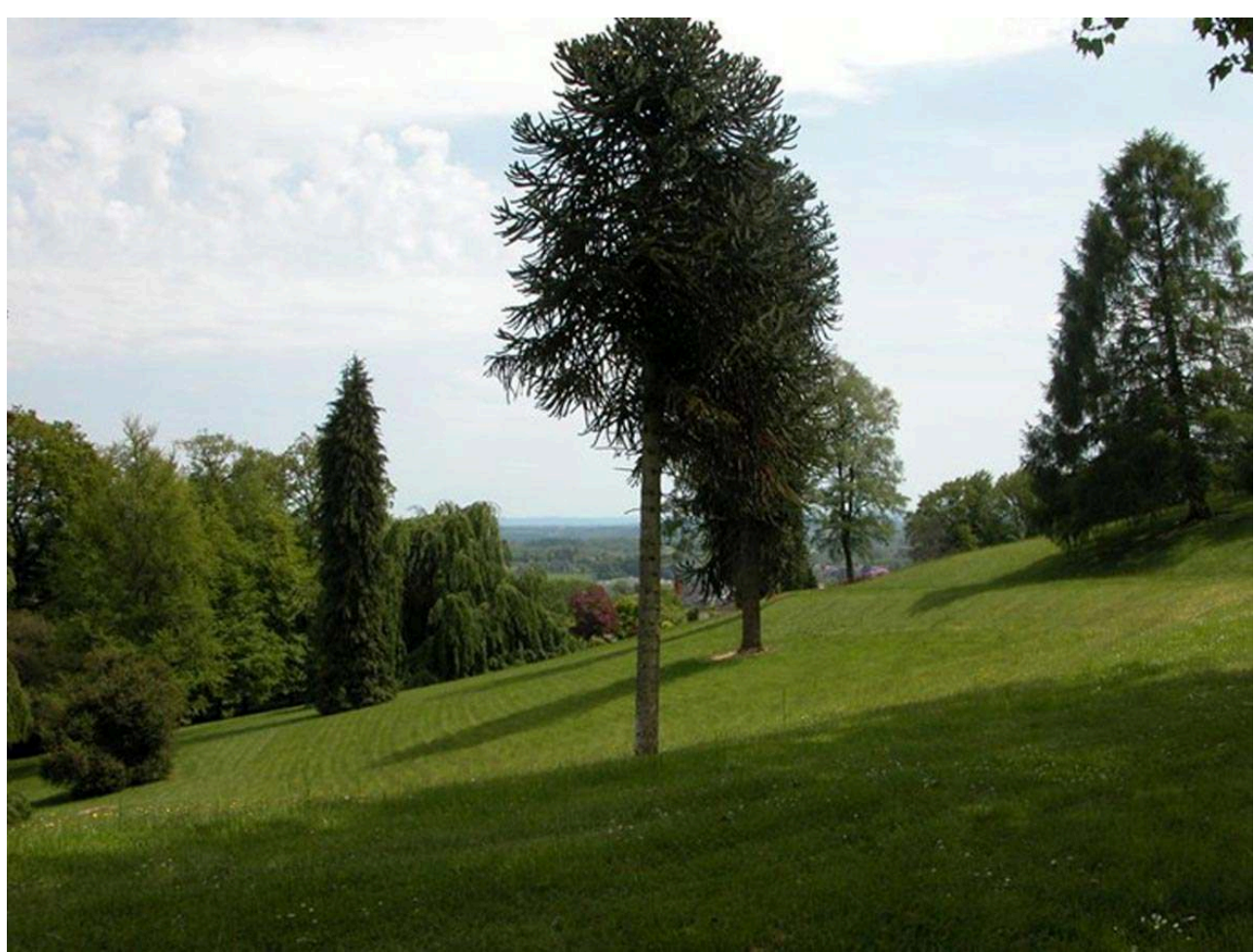

Mont-Méry. Vue du modelé de la pelouse plantée d'essences rares, prise du nord vers le sud Phot. Inv. J.-L. Vey @ Inventaire général, ADAGP, 2005

Les expérimentations des pépinières d'André Laurent sur les conifères vont également dans le même sens, visant à l'introduction et à la culture d'essences rares et bien adaptées aux conditions climatiques de la région. Edouard André qui a dû connaître les monts d'Ambazac ${ }^{28}$ dans leurs caractéristiques géographiques et climatiques a pu influencer de ce point de vue, Théodore Haviland dans le choix de ce site. Mais lorsque ce dernier revient des Etats-Unis avec son épouse américaine, c'est imprégné de la culture et le mode de vie des grandes familles de la côte Est. Homme de l'excellence, il est fort probable que, de concert avec sa femme, il ait fait intervenir, comme pour l'architecture, des praticiens reconnus dans leur cercle de relations américaines.

Aussi ne nous semble-t-il pas déraisonnable d'envisager une attribution du parc de Mont-Méry à Frederick Law Olmsted ou à l'un de ses collaborateurs. Mais il conviendrait de vérifier la pertinence de cette hypothèse par une recherche dans les archives américaines ainsi que par une étude approfondie de la composition et des espèces plantées pour pouvoir établir des comparaisons stylistiques, éventuellement avec les parcs d'Edouard André et avec les créations américaines, ce qui n'a pas réellement été fait en dépit du grand intérêt botanique et artistique de ce parc. Les échanges et les liens étroits entre André, Sargent et Olmsted donnent des indications 
qui orientent la recherche vers les Etats Unis, sans compter l'histoire vraie... des graines de maïs vert ${ }^{29}$.

\section{Deux parcs classés Monuments historiques}

21 Le parc du Reynou, classé Monument historique en 1995 est devenu quelques années plus tard un parc animalier, ce qui n'est pas sans poser un certain nombre de problèmes liés aux aménagements induits par cette activité. L'intérêt paysager qui a été mis en avant lors de l'élaboration du projet et lors de l'ouverture au public a été malheureusement largement perdu de vue.

La situation du parc de Mont-Méry, classé Monument historique en 1991, est par bonheur très différente. Ouvert au public, ce parc est remarquablement entretenu et s'est vu attribuer en 2004 par le ministère de la Culture, le label « jardin remarquable », juste reconnaissance d'une bonne gestion de ce patrimoine vivant. Cependant, certains éléments sont aujourd'hui encore menacés de disparition. C'est le cas par exemple de la délicieuse « Fairy Lodge » qui mériterait une attention urgente (fig. $\left.\mathbf{n}^{\circ} \mathbf{1 4}\right)$. Fleuron du patrimoine des jardins en Limousin, le parc de Mont-Méry est d'un intérêt historique, paysager et botanique qui le place parmi les grands jardins au niveau national. Puisse cette communication contribuer à ouvrir la voie à de nouveaux travaux de recherche et surtout rappeler que pour ne pas mourir, cet ensemble doit être non seulement entretenu mais constamment régénéré.

Figure 14

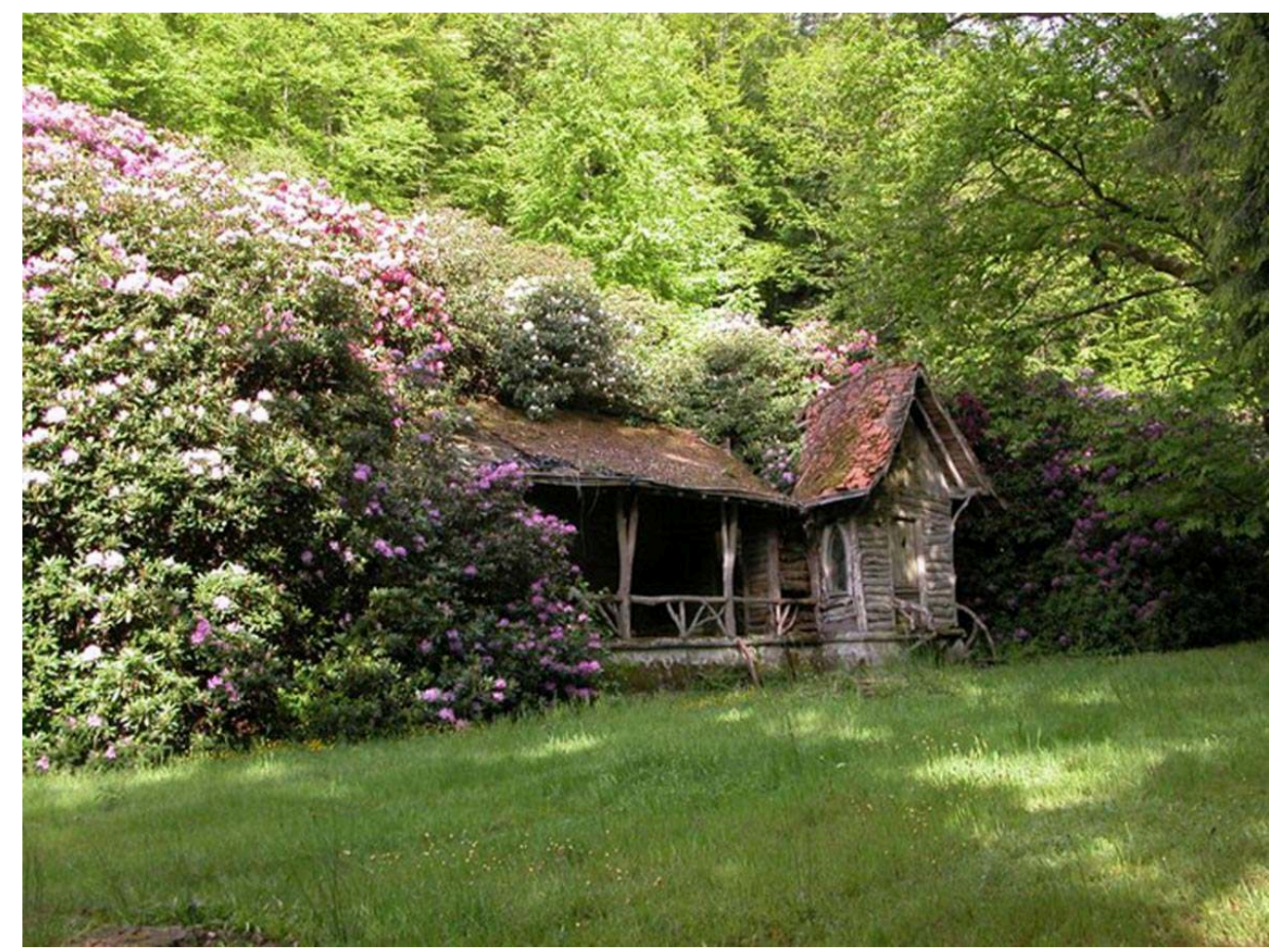

Mont-Méry. «The Fairy Lodge », la maison de la fée, sous son manteau de rhododendrons. Cette construction fragile aurait grandement besoin d'un coup de baguette magique pour une restauration Phot. Inv. J.-L. Vey @ Inventaire général, ADAGP, 2005 


\section{NOTES DE FIN}

1. Albis d', Jean. Haviland. Paris : Dessain et Tolra, 1988.

2. C'est en 1841 que David Haviland quitte les Etats-Unis où il faisait déjà du négoce de porcelaine pour s'installer avec sa famille en France, avec l'idée d'expédier vers New York la porcelaine fabriquée dans les petites manufactures de Limoges. Très vite il fonde ses propres ateliers et le commerce prospère. En 1865, David Haviland associe ses deux fils, Charles et Théodore, au développement de l'entreprise. Charles reste à Limoges et devient le patron de l'usine. Théodore part aux Etats-Unis avec la mission de faire valoir la porcelaine française face à la concurrence anglaise et allemande. La réussite Haviland a été précisément d'imposer cette «French porcelain » sur le marché américain. David Haviland, puis ses deux fils, ont particulièrement marqué le paysage industriel et le développement porcelainier de Limoges. Ils ont été novateurs en tous points par le choix des créateurs de modèles, l'amélioration des machines de fabrication ou des techniques commerciales. Ces hommes ont été aussi des bâtisseurs. L'hôtel particulier de David Haviland construit à l'entrée de l'usine avenue Garibaldi à Limoges est révélateur dans sa conception architecturale d'une culture différente. Cette maison contrastait avec les habitations de l'aristocratie ou de la bourgeoisie de la ville.

3. Le domaine du Reynou est situé sur la commune du Vigen (Haute-Vienne), dans le site inscrit de la vallée de la Roselle. Le château est inscrit MH partiellement, le parc est classé Monument historique depuis 1995. Documentation DRAC du Limousin, dossier d'Inventaire général de la commune du Vigen par Colette Chabrely, 1991; dossier de protection monument historique établi par Annick Tulasne-Moeneclaey pour le château et Colette Chabrely pour le parc; dossier d'étude du parc par Juliette Dessert, architecte paysagiste, 1993. Actuellement, ce parc aménagé en parc animalier est ouvert au public selon les horaires de visite indiqués. Entrée payante.

4. Jacques Chardonne est le pseudonyme littéraire de Jacques Boutelleau, petit-fils de David Haviland.

5. Chardonne, Jacques. Les Destinées sentimentales. 3 tomes : La femme de Jean Barnery Pauline - Porcelaine de Limoges. Paris : Grasset, 1934.

6. Andre, Edouard. Traité général de la composition des parcs et jardins. Jeanne Laffitte. Reimpr. de l'édition de 1879.

7. Benetiere, Marie-Hélène. Jardins, Vocabulaire typologique et technique. Paris : Editions du patrimoine, 2000, p. 41 (coll. Principes d'analyse scientifique).

8. Charles Haviland, né en 1839 à New York a 3 ans lorsque David Haviland s'installe à Limoges. Eduqué dans l'esprit des "quakers », il reçoit une instruction classique au lycée Gay-Lussac de Limoges et en 1864, épouse Marie Guillet, fille d'un magistrat mais aussi descendante des Baignol, une famille de porcelainiers depuis le XVIII ${ }^{\mathrm{e}}$ siècle. Le domaine du Reynou est une propriété agricole de la famille Guillet. La maison, construite autour de 1840, était à cinq travées d'élévation, couverte d'un toit à croupes un peu pentu et à tuiles plates. Une façade ouvre sur une cour des communs comme en témoigne le cadastre napoléonien, l'autre, orientée au sud-ouest, domine un paysage bocager vallonné. Des caractères de construction plutôt récurrents pour une maison de maître et une vue sur une campagne agricole plaisante que Charles Haviland saura parfaitement utiliser.

Après la mort de Marie Haviland en 1873, Charles Haviland épouse en 1877 Madeleine Burty, fille du critique d'art Philippe Burty. Cette période coïncide avec les succès de l'exposition de Philadelphie en 1876 puis de Paris en 1878. Sa vie est alors plus parisienne que limousine. Les modèles de porcelaine créés dans ces années-là bénéficient de l'influence culturelle de la capitale notamment avec Félix Bracquemont. Ces influences artistiques parviennent jusqu'au Reynou.

C'est en 1876-77, selon les matrices cadastrales, que l'ancienne maison Guillet est agrandie de 
deux pavillons disposés de part et d'autre du corps d'origine ce qui la transforme en petit château au répertoire ornemental classique. Une corniche moulurée et un fronton triangulaire sont ajoutés à l'ancienne maison pour harmoniser l'ensemble. Ces éléments seront supprimés lors des ajouts suivants réalisés sur un mode plus pittoresque.

Cette construction devient grandiloquente avec l'édification de l'aile nord en 1894 et de l'aile sud en 1895 d'après les dates portées sur les plans de ces deux adjonctions. La construction de l'écurie, de la sellerie ainsi que d'une remise, s'échelonnent entre 1890-1893 et 1894-96. Enfin les derniers travaux concernant la création du grand salon sur deux niveaux de l'ancienne maison sont de 1907. Les élévations pour aménager les grandes baies vitrées de la façade antérieure sont de 1908-1909. Ces derniers travaux sont commandés par Louis Albert Camu, époux de Jeanne Haviland ; Charles avait légué cette propriété à sa fille en 1907. L'ensemble est une illustration de l'éclectisme pittoresque avant d'être de ce qu'on est tenté d'appeler aujourd'hui une « construction évolutive»!

9. Valiere, Nathalie. Un Américain à Limoges : Charles Edward Haviland (1839-1901). Tulle : Société Historique et Régionaliste du Bas-Limousin, 1992.t. 17 des Mémoires d'Histoire Régionale des Editions Lemouzi, $240 \mathrm{p}$.

10. Madame Edmée Haviland, petite-fille de Charles Haviland, rencontrée en 1993 à Paris, n'avait pas de précision vraiment historique à apporter. Elle avait gardé du Reynou le souvenir du bruissement des râteaux passés tous les matins sur la terrasse par le « bataillon de jardiniers ». La domesticité employée à l'entretien du château et du parc était selon d'autres sources orales de 27 personnes, ce qui, aux environs de Limoges, représente des moyens qui ne devaient pas avoir d'équivalent.

11. Les photographies anciennes du Reynou proviennent de la collection de Monsieur Charles Dupont, propriétaire en 1990. Les descendants de Monsieur Dupont n'ayant pu être joints, l'autorisation à reproduire ces documents n'a pu leur être demandée.

12. Les catalogues de vente édités en 1922-23 par l'hôtel Drouot lors de la vente de ses collections n'inventorient pas moins de 6000 estampes japonaises, de la porcelaine de Chine, des objets d'Extrême-Orient et de très nombreux tableaux.

13. André Laurent, pépiniériste et créateur de parcs est assez peu connu des historiens des jardins. Selon sa nécrologie, il est né en 1825. Apprenti à 12 ans, il était connu sous le nom de la «main d'or» dans la région angevine où il apprit l'horticulture, la multiplication, l'hybridation, le métier de pépiniériste, le tracé et la plantation des parcs et jardins. Aussi lorsque vers 1846 l'horticulteur limousin Peyte recherche auprès de la maison Leroy d'Angers un homme (...) capable de rénover son établissement à la manière angevine c'est André Laurent qui est proposé. Il arrive à Limoges en 1848 et devient directeur de la maison Peyte, puis assez vite, il installe plusieurs pépinières. Il fonde en 1860 l'Ecole d'horticulture de Limoges et en devient directeur. En 1861, le comte de Choulot, paysagiste, qui avait par ailleurs l'occasion de travailler avec la maison Leroy d'Angers, lui confia la plantation du parc de la Chabroulie aux environs de Limoges. Un plan d'exécution de Choulot porte en marge la mention «Laurent jardinier ». En 1883, en association avec Henri Gérardin, il crée la pépinière de la Jonchère dans les monts d'Ambazac, à une quinzaine de kilomètres de Mont-Méry. Les expérimentations de ces deux hommes visant à introduire dans la région de nouveaux arbres, notamment des conifères, et à observer leur développement sur le sol limousin sont un élément important qui a favorisé la réalisation de nombreux petits parcs paysagers autour de Limoges. Ces créations sont repérables à partir des années 1877-78. Des plans portant sa signature sont datés des années 1885-90. Les différents plans comparés les uns aux autres sont assez semblables, ce qui les distingue vient plutôt du site lui-même. Les allées courbes, les vues bien marquées, les éléments décoratifs artificiels tels que les enrochements procèdent pour les dessins d'André Laurent de l'utilisation appliquée des principes du paysagiste Edouard André dont il était le parfait émule. André Laurent se distingue plus spécifiquement par sa très bonne connaissance des arbres et des végétaux et par l'utilisation 
qu'il en fait dans ses projets. (Chabrely, Colette. Plans et parcs du comte de Choulot en HauteVienne. Polia. Revue de l'art des jardins, 2004, nº 1, p. 79 à 93).

14. Taboury, M.-F. Nécrologie d'André Laurent. Bulletin de la Société d'horticulture et d'arboriculture de la Haute-Vienne, 1907, t. 45, p. 8 à 12.

15. Le parc de Plantadis situé sur la commune de Saint-Martin-Terressus (Haute-Vienne) a été entièrement dévasté par la tempête de 1999. (propriété privée, ne se visite pas).

16. Celui de Crochat est situé sur la commune de Limoges. (propriété privée, ne se visite pas).

17. L'excursion printanière des «Amis des Fleurs » au château du Reynou. La Vie limousine. 25 juin 1931.

18. Racine, Michel. Jardins « au naturel». Rocailles, grotesques et art rustique. Actes sud, 2001. p. 96 à 99 .

19. Le domaine de Mont-Méry est situé sur la commune d'Ambazac (Haute-Vienne) dans le site inscrit du Mont Gerbassou. Le château et le parc sont classés Monuments historiques depuis 1991. Dossier documentaire établi par Annick Tulasne-Moeneclaey en 1982, revu par Bénédicte Chantelard en 1988 pour l'architecture et par Marie-France Morel et Françoise Chaput, inspecteur des sites, DIREN, pour le parc. En 2004, il a reçu le label «jardin remarquable » du ministère de la Culture et de la Communication. Propriété privée, il est ouvert au public les dimanches de juin à septembre, toute l'année sur demande. Entrée payante.

20. Voir la note 2 .

21. Le château de Mont-Méry, situé sur les flancs sud et est du Gerbassou, porte le monogramme de Théodore Haviland et la date d'achèvement de la construction 1889. Le parti stylistique qui est choisi par Théodore est celui des maisons à la mode, nouvellement construites au nord-est des Etats-Unis, autour de Boston, Newport ou Long Island, commanditées par une haute société, aspirant à un nouveau mode de vie. La silhouette générale du château de Mont-Méry est celle de ces maisons-là ; l'examen dans le détail des différents éléments constitutifs renvoie aux styles du vieux continent dans leur diversité. En effet, ces maisons nord-américaines sont faites d'une juxtaposition de références au vocabulaire ornemental des modèles européens. Il en est ainsi du château de Mont-Méry. La façade principale emprunte notamment à elle seule à l'art roman, à la Renaissance (porte d'entrée), à l'architecture vénitienne (galerie en triforium), mais aussi au classicisme. Une partie de la façade ouest tire son parti architectural du style colonial américain. Cette ornementation éclectique cache un parti architectural simple, d'une rationalité moderne. Pour l'ensemble de cette construction, l'emploi de la pierre de taille en granite limousin plutôt que la brique est la preuve qu'il y a une adaptation locale de l'esprit américain.

Selon les notes transmises par la propriétaire lors de la constitution du dossier de protection au titre des Monuments historiques, cette construction serait de Richard Morris Hunt. Le nom d'un certain James, peut-être chef d'atelier, apparaît pour un avant-projet. Apparemment il n'y a pas de source écrite. Ce qui est plus certain c'est que les travaux ont été suivis par l'architecte Menissier de Limoges. La contribution plus ou moins directe de Hunt semble tout à fait possible d'autant que ce dernier a construit les stands d'exposition des porcelaines Haviland aux Expositions universelles de Philadelphie en 1876 et de Paris en 1878.

Richard Morris Hunt, formé aux Beaux-Arts et élève de Lefuel et Théodore Haviland ont acquis l'un et l'autre, par des cheminements inverses, la double culture franco-américaine. Mont-Méry serait en quelque sorte la construction du retour. Retour de Théodore, retour des modèles.

Outre les références architecturales énumérées précédemment, ce qui semble le mieux qualifier l'ensemble du projet c'est la totale prise de possession du site. C'est aussi ici ce qui est le plus réussi. Toutes les pièces principales sont largement ouvertes sur le parc, le paysage et les lointains. La tourelle disposée au sud, totalement ajourée à chaque niveau, en est le meilleur exemple. (Loyer, François. Histoire de l'architecture française. De la Révolution à nos jours. Paris : Editions du patrimoine, 1999, p. 388, n. 269).

22. La maison de la fée. 
23. Dossier documentaire du service des Monuments Historiques, DRAC du Limousin.

24. Florence André, historienne des jardins, venue visiter ce parc en 2002, n'avait pas connaissance dans les archives d'Edouard André, son arrière grand-père, de document concernant ce parc.

25. Edouard André (1840-1911) Un paysagiste botaniste sur les chemins du monde. Sous la direction de Florence André et Stéphanie de Courtois. Paris: les Editions de l'Imprimeur, collection Jardins et paysages, 2001, $235 \mathrm{p}$.

26. Andersen, Phyllis. «Mon cher ami » : l'amitié entre Charles Sprague Sargent et Frederick Law Olmsted. Edouard André (1840-1911) Un paysagiste botaniste sur les chemins du monde. Paris : les Editions de l'Imprimeur, collection Jardins et paysages, 2001, p. 83-92.

27. Voir supra note 19.

28. La famille Pouquet, propriétaire du domaine de Trasforêt, situé tout près de Mont-Méry, a invité la famille du musicien Auguste Franchomme à s'y replier pendant les événements de 1870 . Franchomme était le beau-père d'Edouard André. Les liens que les familles pouvaient entretenir sont avérés.

29. Des informations orales ont été recueillies auprès de Madame Edmée Haviland, à Paris en 1993, de Madame Mary Mallet au Bois des Moutiers à Varengeville, en 1998 et de Monsieur Jean d'Albis à Limoges vers 1990. Que la mémoire de ces trois personnes qui m’ont reçue soit ici honorée.

A Mont-Méry, Madame Laure de Pourtalès m'a donné accès à toutes les informations en sa possession, qu'elle en soit tout particulièrement et vivement remerciée.

\section{RÉSUMÉS}

Les parcs du Reynou et de Mont-Méry en Limousin, anciennes propriétés des porcelainiers Haviland, présentent l'un et l'autre un grand intérêt paysager et botanique. Dans les deux cas, l'attribution de la création est incertaine. Cet article propose pour le Reynou une analyse de documents figurés anciens permettant de préciser la chronologie des travaux du château et du parc. Pour Mont-Méry il s'agit de poser de nouveaux jalons pour une étude plus approfondie des sources et de la composition des jardins afin d'envisager de nouvelles pistes pour leur attribution.

The Reynou and Mont-Méry parks near Limoges originally belonged to the city's porcelain manufacturers, the Havilands. Both parks are of considerable interest in terms of their landscaping and their botany. In both cases, there is some uncertainty as to the identity of their designers. This article offers an analysis of the graphic representations of the Reynou park, clarifying the chronology of the creation of the chateau and its park. For the Mont-Méry park, the aim is to offer some guidelines for further research in the source material and on the design of the park itself, perhaps allowing for the designer to be identified.

\section{INDEX}

Keywords : Landscaping, botany

Mots-clés : Haviland, porcelaine, Limousin, Frederick Law Olmsted, Mont-Méry, Le Reynou, André Laurent, Gabriel Lecardeur, sites protégés, monuments historiques, Edouard André, arboretum, rocaille 
AUTEUR

COLETTE CHABRELY

Ingénieur d'études. Inventaire. Région Limousin. colette.chabrely@culture.gouv.fr 